\title{
Solution of the problem of recultivation of sludge sites at the oil fields by electroosmosis
}

\author{
Evgeny Porsev ${ }^{1, *}$, Boris Malozyomov ${ }^{1}$ and Marina Rozhkova ${ }^{1}$ \\ ${ }^{1}$ Novosibirsk State Technical University, 20, Karl Marks Av., Novosibirsk, 630073, Russia
}

\begin{abstract}
The problem of reclamation of sludge pits in oil fields is still being solved using the resourceintensive method. An accelerated technology of sludge dewatering by electroosmotic action is proposed. The concept of specific energy consumption for mass transfer is introduced. To eliminate the influence of relaxation of gradient fields, it is proposed to change the parameters of electrical energy. A graphicanalytical method for selecting the power of an energy source is proposed. The article shows that electroosmosis can be the optimal physical process for dewatering drill cuttings. To reduce the time of the process of reclamation of sludge pits, it is advisable to eliminate the relaxation of various force gradient fields by changing the parameters of the electricity supplied to the electrodes. Recommendations are given that the technological cycle of reclamation should be carried out in one summer season, during which, using electroosmosis, reduce the moisture content of the sludge and the concentration of chemical pollutants to an acceptable level, and then sow the barn with plants and thereby return the area to normal use.
\end{abstract}

\section{Introduction}

Slurry pits are pits about $50-100 \mathrm{~m}$ in diameter, sometimes smaller, they are created near boreholes to store drilling waste: waste solutions, with or without oil, crushed rock, clay, water, various chemicals. Each barn stores about 500 cubic meters of drilling waste per well. Drilling waste, diluted with water, is a creamy mass in terms of viscosity, to a certain extent toxic.

To eliminate this threat to the environment, the sludge pits are reclaimed during well construction. There are several ways of recultivation: backfilling with sand, natural evaporation, thermal and chemical treatment, injection into dry absorbing strata, «squeezing» into narrow trenches and removal to evaporation fields. All of them are very costly, in terms of both time and finance.

To fill up one barn, it is necessary to bring 4-5 thousand cubic meters of sand, spending a lot of money on all the work to liquidate the barn. At the same time, in order to bring sand from other places, it is necessary to destroy the forest and take sand from under it, or get it from a lake or swamp.

The purpose of the work from the point of view of the criterion of public utility is to accelerate the process of reclamation of sludge pits, to save primary energy resources for the process, and to increase the environmental safety of oil fields.

To achieve this goal, it is necessary to solve the following tasks:

- to find the best way to dehydrate the sludge;

- to calculate the technological cycle of the reclamation system on a new basis;
- to develop conceptual diagrams of technology and an electrical diagram of electroosmotic reclamation of sludge pits of oil fields.

Analysis of the paper's and patent's situation in the field of reclamation technology for the period from 1975 to 2008 in order to determine the trend in the development of technology showed (Figure 1 and Figure 2):

1. The distribution of the frequency of repetition of filing applications over the years in the study period is uniform, which indicates that the problem of reclamation has not been resolved.

2. Analysis of the frequency of repetition of filing applications for patents in the study period suggests that the problem has not been resolved, and a slight increase in the number of filings in recent years indicates an increase in the relevance of technical solutions related to land reclamation by technical means.

3. Obviously, the problematic situation is that in this area there are no radical revolutionary solutions that would help solve the problem in traditional ways.

4. We assumed a working hypothesis of the possibility of solving the above problem by an electrotechnological method - the use of electroosmotic dewatering.

Based on the analysis of the patent situation, we can say that until now electroosmosis has not been used for dewatering sludge pits, despite the widespread use of this technology in other fields of technology. In our opinion, the reason is the difficulty of predicting the result and the lack of practical recommendations. At the same time, electroosmosis allows one not only to reduce

\footnotetext{
Corresponding author: porseveg@ mail.ru
} 
the moisture content of the sludge, but also to solve other problems.

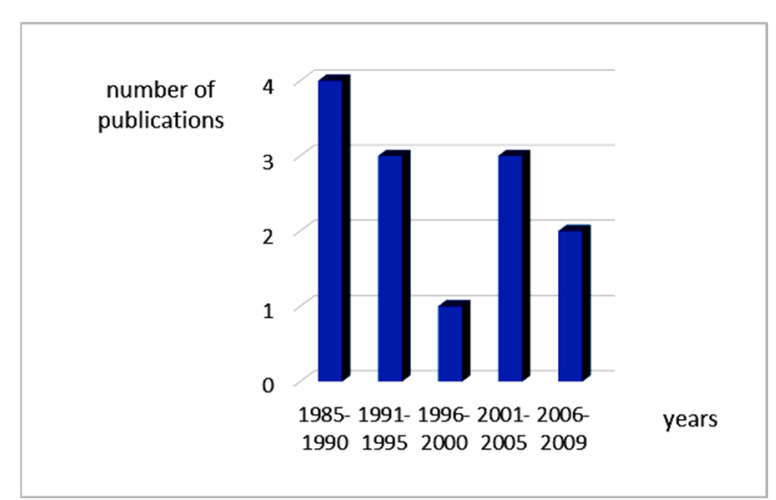

Fig. 1. Histogram of the number of received patents and applications filed by year.

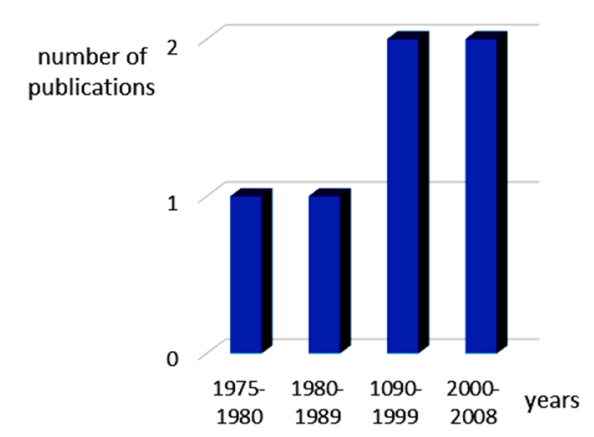

Fig. 2. Histogram of the number of published scientific articles by year.

The trend in the development of technology based on the results of the analysis of published scientific articles over the years allows one not only to lower the moisture content of dispersed material, but also to remove hardness salts from it together with moisture, which also contributes to the acceleration of the reclamation process.

\section{Mathematical description of a model}

Wiedemann carried out the first quantitative studies of electroosmosis in 1852. He showed that the volumetric velocity $V$ of electroosmosis is proportional to the current strength $I$ with other fixed parameters, and the ratio $V / I$ does not depend on the cross-sectional area and thickness of the diaphragm [1]. The first quantitative characteristic of the process was the volumetric velocity, which, according to Lomize [2], shows the consumption of electroosmotically transferred water per unit of the total cross section of the dehydrated material occupied by the skeleton and pores. The speed is expressed in meters per second. With the deepening of knowledge in the field of colloidal chemistry and dispersed systems, the potential $\xi$ [3] (Helmholtz and Perrin), measured in volts (V), was taken as the main indicator of the electrokinetic properties of a material. This indicator reflects the essence of physical phenomena, but in practice it is inconvenient, since for its determination it requires accurate measurements of the dielectric constant and viscosity.

In 1940, B.F. Reltov proposed to introduce a new indicator to determine the efficiency of the process - the coefficient of electroosmosis - $K_{e}$ [4]. The electroosmosis coefficient determines the effect of solid and liquid components on the volumetric velocity of electroosmosis and is equal to the fictitious speed of water movement under the action of an external electric field $E$ with a field strength $\mathrm{V} / \mathrm{cm}$. The value of the coefficient is defined as:

$$
K_{e}=\frac{\rho \xi \varepsilon}{4 \pi \eta}
$$

where $K_{e}$ is, firstly, the coefficient of proportionality between $V_{e}$ and $E$, secondly, the integral characteristic:

$$
V_{e}=K_{e} \cdot E_{;}
$$

At the same time, it is an auxiliary characteristic for determining the volumetric velocity. This was further developed in the coefficient of electroosmosis by current - $K_{e i}$ ( $\left.\mathrm{m}^{3} / \mathrm{As}\right)$, proposed by R.S. Ziangirov [5]. In contrast $K_{e}$ is the coefficient of proportionality between $V_{e}$ - the volumetric velocity and $\sigma$ - the current density:

$$
V_{e}=K_{e i} \sigma
$$

The electroosmosis coefficient is related to the current electroosmosis coefficient by the dependence:

$$
K_{e}=\gamma K_{e i}
$$

where $\gamma$ is the specific electrical conductivity of the processed material, $\mathrm{Ohm}^{-1} \mathrm{~m}^{-1}$. Both of these coefficients make it possible, when designing, to determine the liquid yield when operating on direct current and the power consumption in amseconds, given the required volume of water. However, when working on $\mathrm{AC}$ or $\mathrm{AC}$ asymmetric current, this is difficult.

In connection with the need to perform work on an alternating asymmetric current, it became necessary to evaluate the energy process not by traditional coefficients $K_{e i}$ and $V_{e}$, but by a characteristic that takes into account all the consumed electricity. It was proposed to evaluate the energy efficiency in terms of specific energy consumption for liquid separation:

$$
w_{e}=W / M ; \quad[\mathrm{kWh} / \mathrm{kg}]
$$

where $W$ is the amount of electricity spent on the dehydration process, $\mathrm{kWh}$;

$M$ - mass of water released by electroosmotic pressure, $\mathrm{kg}$.

It is also possible to estimate in specific power:

$$
P_{e}=\frac{w_{e}}{\tau}=\frac{W}{M \tau} ; \quad[\mathrm{kW} / \mathrm{kg}]
$$

where $\tau$ is the processing time, hours. 
Later, Lockhart adopted the same system for evaluating the energy of the process [6]. The advantage of the assessment system adopted by us is that it is easy to calculate the required power and operating current of the installation knowing the specific energy consumption, specifying the required dehydration depth, and processing time. Obviously, in each case, an experimental determination of the specific energy consumption is required.

Taking the specific energy consumption for water release as the criterion for optimizing the electroosmosis process, we will also obtain a research method based on taking into account the real energy consumption of the technological process, and not only the physical properties of one dispersed material. A device designed to work according to this technique should have its own characteristics: the ability to fix water consumption, energy consumption, and the mass of the processed material. Knowing the water consumption and energy consumption for the process, it is possible to calculate the specific energy consumption for water extraction:

$$
w=W / M_{x} \quad[\mathrm{kWh} / \mathrm{kg}],
$$

where $W$ is the energy consumption for the process, $\mathrm{kWh}$;

$M_{x^{-}}$mass of released water, $\mathrm{kg}$.

Further, knowing the initial moisture content of the material $-B_{1}$, and setting the required final $B_{2}$, we calculate the mass of water that must be separated from the material - $M_{x}$, using the well-known equations of the material balance of the separation process [7].

For the technology of dehydration by electroosmosis, subject to the water content in the withdrawn liquid fraction $B_{x}=1$, the following can be obtained:

$$
\left.\begin{array}{c}
M_{1}=M_{x}+M_{2} \\
M_{1}\left(1-B_{1}\right)=M_{x}\left(1-B_{x}\right)+M_{2}\left(1-B_{2}\right) \\
M_{1} B_{1}=M_{x} B_{x}+M_{2} B_{2} \\
M_{x}=M_{1} \frac{B_{2}-B_{1}}{B_{2}-B_{x}} \\
M_{1}=M_{x}+M_{2} \\
M_{1}\left(1-B_{1}\right)=M_{2}\left(1-B_{2}\right) \\
M_{1} B_{1}=M_{x} B_{x}+M_{2} B_{2} \\
M_{x}=M_{1} \frac{B_{2}-B_{1}}{B_{2}-B_{x}}
\end{array}\right\}
$$

where $M_{1}, M_{x}, M_{2}$ - masses of the initial mixture, the liquid fraction removed from the mixture and obtained as a result of processing wet dispersed material, $\mathrm{kg}$;

$B_{1}, B_{x}, B_{2}$ - water content in the initial mixture, in the liquid fraction and the resulting dispersed material, p.u.

Next, you can determine the total energy consumption of the process:

$$
W=w M_{x}[\mathrm{kWh}]
$$

\section{A physical model of the installation}

Knowing the specific electrical resistance of the material and the voltage at the electrodes, it is possible to determine the operating current of the installation and the processing time.

Obviously, to determine the specific energy consumption, it is necessary to experimentally determine this value on an electroosmometer (Figure 3).

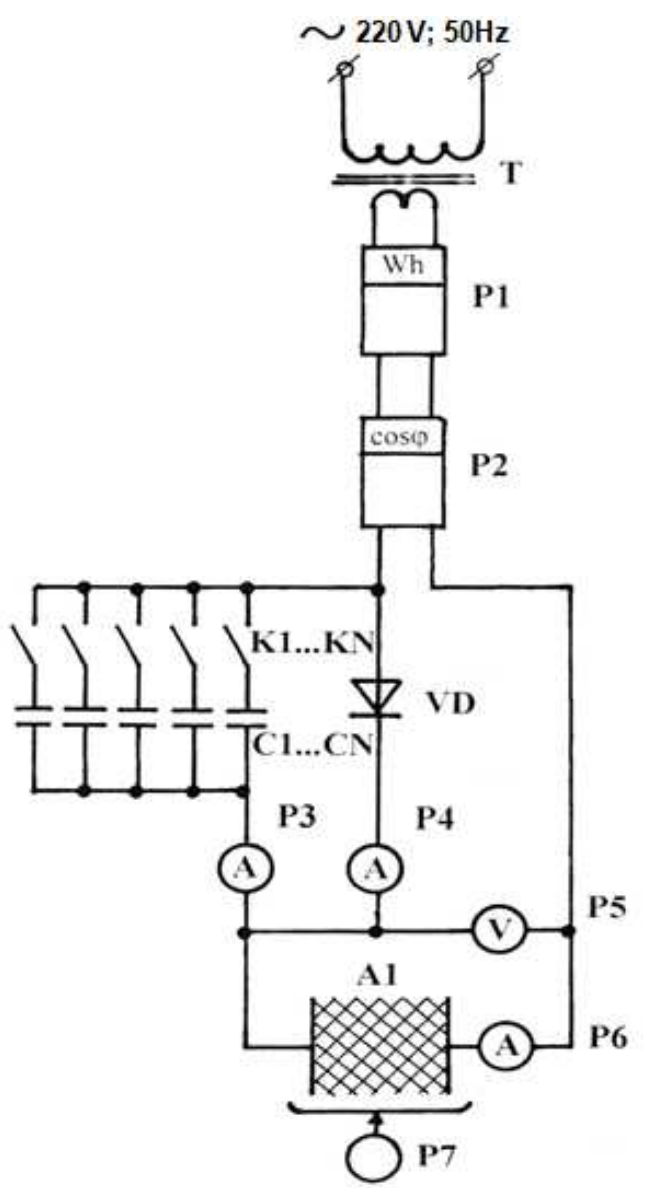

Fig. 3. Schematic diagram of the electroosmometer: T transformer, P1 - electricity meter, P2 - phase meter, VD power silicon valve, $\mathrm{Cl} \ldots \mathrm{CN}$ - capacitors, $\mathrm{K} 1 \ldots \mathrm{KN}$ - toggle switches, P3, P4, P6 - ammeters, P6 - voltmeter, A1 electroosmometer, $\mathrm{P} 7$ - platform scales.

Due to the fact that almost all dispersed soil materials contain mineral particles as a dispersed phase, and water as a dispersion medium, the process of electrokinetic energy and mass transfer in them has its own characteristics, namely:

a) Heteroporosity of the dispersed phase, that is, the inhomogeneity of capillary sizes, which depends on a large scatter of particle sizes, leading to excessive consumption of electrical energy for heating the dispersion medium in large capillaries.

b) The multicomponent composition of the electrolyte composition of the dispersion medium, that is, the presence of indifferent and non-indifferent electrolytes in the solution, leading, on the one hand, to a decrease in the electrokinetic potential as a result of an increase in the concentration of counterions with an increase in the concentration of indifferent electrolytes and, on the other hand, the ability of electrolyte ions to 
complete the crystal lattice of the dispersed phase, leading, ultimately, to a drop in the electrokinetic potential.

c) The difference between the $\mathrm{pH}$ of the medium and $\mathrm{pH}$ from neutral can strongly affect the electrokinetic potential of dispersed particles, since "hydrogen and hydroxyl ions have a high ability to be adsorbed. The first is due to the small radius, which allows them to come close to the surface of the solid phase. The latter are due to the large dipole moment" [6]. The deviation of the $\mathrm{pH}$ to the acidic or alkaline side for the dispersion medium of soils is characteristic.

d) Dependence on the concentration of the dispersed phase - when the colloidal system is diluted, the electrokinetic potential should increase, since the thickness of the electric double layer increases as a result of a decrease in the concentration of counterions in the solution. At the same time, upon dilution, desorption of the potential-determining ion from the surface of the dispersed phase can be observed, which should lead to a drop in the electrokinetic potential. The phenomenon is typical of electrophoresis in highly diluted wastewater.

e) Dependence on the processing temperature - with an increase in temperature, the $x$-potential should increase due to an increase in the intensity of the thermal movement of counterions and an increase in the thickness of the electric double layer. However, at the same time, desorption of potential-determining ions can also increase, and the x-potential decreases.

f) Instability of the electrophysical and electrochemical properties of the dispersed phase, logically deduced from the physical nature of the particles of the dispersed phase and the chemical activity of the dispersed medium.

Thus, the main feature of the process of electrokinetic processing of dispersed materials is the relatively high specific energy consumption for the process, the dependence of specific energy consumption on a large number of uncontrollable and difficult to control factors due to the inadequacy of existing theory and technology.

\section{Development of the theory of electroosmosis}

The distribution of energy fluxes, which characterizes thermal diffusion and electrodiffusion phoretic processes, suggests that only part of the electrical energy will be lost, while some will participate in beneficial mass transfer in the form of thermal and moisture conductivity, ionosmosis and iontophoresis. The distribution of energy flows of other diffusion processes is similar [5].

The electrokinetic process in the energy aspect is a system of a number of physical, electrophysical, electrochemical, thermal diffusion and diffusion processes. The group of electrophysical and electrochemical processes is characterized by polarization at the molecular and ionic levels and electrolysis, which, when operating at direct current, inevitably lead to excessive losses. At first glance, the simplest solution for removing polarization is to switch to an alternating asymmetric current.

During electroosmosis, a number of researchers note the "orientational ordering of particles" and the formation of "chain structures" under the influence of polarization forces. In addition, with a variable asymmetric feeding, a decrease in the weakening field, the effect of relaxation of the diffuse layer and, as a consequence, an increase in the water yield at the same energy consumption should affect.

In this case, in order to reduce dielectric losses, it is advisable to match the relaxation time of the DEL of the material being processed and the frequency of the change in the electric field; the period of oscillations of the electric field strength should not exceed the relaxation time. Any polarization process is characterized by a finite relaxation time, so that at sufficiently high frequencies, when the field period is commensurate with the relaxation time, the phase shift between the polarization and the field becomes noticeable, which manifests itself in dielectric losses. The relaxation time according to the theory [15] for electrolytic polarization, that is, for the relaxation of DES, lies in the range of 10-2 ... 10-4 sec. Experiments have shown that the relaxation of DES affects the frequencies $f=20 \ldots 320 \mathrm{~Hz}$ least of all.

During the operation of installations for electroosmotic dehydration of wet dispersed materials, researchers observed «heating and overdrying of the soil adjacent to the anodes» [10]. The drying of the material in the anode region under the influence of electroosmosis leads to a significant increase in the specific resistance of the material and to an increase in the voltage drop in the region adjacent to the anode. An increase in the voltage drop, in turn, leads to an increase in heating and an even greater overdrying of the material. Thus, near the anode, a progressively increasing process of overheating and overdrying of the material is possible, limiting the capabilities of the electroosmotic installation.

Hence, it can be concluded that the specific energy consumption increases as the anode zone is drained. Switching the positive pole of the source from the anode to the anode located on the line connecting the most distant anode to the cathode should provide the most advantageous operating mode for each anode, since switching can be performed before the appearance of a dried zone, thereby dramatically reducing heat losses in the anode zone. In this case, it is advisable to additionally measure the electric current through the material, and connect the anodes to the power source sequentially in the direction of the electric field, and disconnect the first anode from the positive pole of the power source and connect the next one taking into account the measured value of the electric current. The setting of the current relay should be such value at which the specific energy consumption will be at an acceptable level from the point of view of the economy [7].

From the theory of continuum mechanics for a finite volume, it follows that electroosmosis is a process with diffusion, with the first component (dispersion medium moisture) moving in one direction relative to the initial volume, the second (dispersed phase - solid dielectric) in 
the other. Consequently, when studying the motion of multicomponent mixtures, it is necessary to combine the laws of continuum mechanics with the laws of physics and chemistry to change the mass of a mixture component per unit time per unit volume.

Consequently, the phenomenon of electrokinetics cannot be considered in isolation from the mechanics of a continuous medium, especially when working with closed volumes of processed materials. It follows from theory that the mass of each of the components in local volumes is subject to change and therefore electrokinetic (electroosmosis coefficient), electrical (specific electrical conductivity) and electric power (specific energy consumption for moisture release) characteristics of materials. It should also be noted that the theory of a continuous medium takes into account the motion of all components relative to the initial volume at the same time - the principle of superposition. This was not taken into account in previous works on electrokinetics due to the fact that processes were considered in unlimited volumes that occur only during soil dehydration [4]. When dispersed materials are dehydrated by electroosmosis in closed volumes, there is a continuous change in the physicochemical characteristics of the material, which is expressed in an increase in the specific electrical resistance of the material in the anode zone and a decrease in the cathode zone. As you know, there is a stable interdependence between specific electrical resistance and energy consumption. In practice, dehydration of the anode zone leads to a loss of electrical conductivity of the anode zone, to an increase in specific energy consumption and, ultimately, to the termination of the dehydration process until satisfactory characteristics of the processed materials are obtained.

The greatest influence on the growth of energy consumption in the process of electroosmotic dehydration in a closed volume is exerted by the accelerated drying of the anode zone and a jump in heat losses on the increased electrical resistance of the anode zone. The relationship between the electrical resistance and moisture content of the material is obvious and experimentally proven. Moreover, the sharply identified drained anode zone is not large - its thickness is usually within the range of $0.05 \ldots 0.1 \mathrm{~m}$, but sufficient to interrupt the electrical circuit. In this case, the main part of the supply voltage falls to the dried anode zone, which has a high electrical resistance and an insignificant part on the entire remaining volume of the material. In addition, the field strength in the material turns out to be insufficient for the separation of loosely bound water and the electroosmosis process does not take place. Electric energy is consumed only for heating the drained anode zone. In order to move the loosely bound water, it is necessary to re-create the electric field strength in the dehydrated mass above the threshold. This can be achieved in several ways: using the anode effect, switching the power supply from the anode to the anode - "running pulse", intermittently operating the power source.

At the same time, experts have the opinion that electroosmosis should be carried out on direct current the logic of the work of the electroosmotic pressure forces speaks of this, no one paid attention to the possibility of other relaxation phenomena occurring simultaneously with electroosmosis. Taking into account the phenomena occurring along the way with electroosmosis leads to the idea of carrying out dehydration on an alternating asymmetric electric current. This allows removing the negative effect of the electric polarization of the electrodes [7], the effect of the relaxation of the hydrostatic polarization of the dispersion medium is removed by pulsed power supply [4]. In addition, the effect of relaxation the forces of thermal and moisture conductivity are removed by applying power supply "running impulse" [2]. In total, these three methods give a gain in electricity consumption compared to electroosmosis with direct current up to 10-11 times.

\section{Conclusion}

It has been established that electroosmosis can be the optimal physical process for dewatering drill cuttings. To reduce the time of the process of reclamation of sludge pits, it is advisable to remove the relaxation of various force gradient fields by changing the parameters of the electricity supplied to the electrodes. It is assumed that the technological cycle of reclamation should be carried out in one summer season, during which, using electroosmosis, reduce the moisture content of the sludge and the concentration of chemical contaminants to an acceptable level, and then sow the barn with green manure and thus return the territory to agricultural use.

\section{References}

1. G.Ja. Ivanov, B.V. Malozyomov Reliable power saving electric drive of wide application, (KORUS, 2005)

2. Yu. P. Filyushov, P.V. Zonov, B.V. Malozemov Energy efficient control of an alternating current machine (The Polzunovsky Herald, 2011)

3. Yu. Loshkarev, A.S. Chernyshov Unbrakable control. Features of methods of nondestructive testing (Actual problems of power engineering of agrarian and industrial complex Materials of IV International scientific and practical conference, 2013)

4. O.A. Taranina, V.S. Burkat Ecological systems and devices 6 (2017)

5. C. Tomescu IOP Conf. Ser.: Earth Environ. Sci. 44, 8 (2016)

6. M.A. Gasanov IOP Conf. Ser.: Earth Environ. Sci. 50, 7 (2017)

7. E.A. Ivantsova IOP Conf. Ser.: Earth Environ. Sci. 224, 6 (2019) 